\title{
The merging of two co-rotating vortices: a numerical study
}

\author{
Ch. Josserand *, M. Rossi \\ Laboratoire de Modélisation en Mécanique, Université de Paris VI, 4, place Jussieu, 75252 Paris cedex 05, France
}

Received 23 February 2006; received in revised form 5 October 2006; accepted 27 February 2007

Available online 3 April 2007

\begin{abstract}
The merging of two-dimensional co-rotating vortices is analysed through direct numerical simulations at large Reynolds numbers. It is shown how the Reynolds number affects each of the three phases that characterise this phenomenon. In the first phase, we examine the merging onset and focus on its definition. During the second rapid phase, the contributions of various flow regions upon the dynamics of a vortex are quantitatively studied. These regions are respectively the companion vortex, the filaments and an intermediate zone between vortices and filaments. The third phase is interpreted in terms of an advection diffusion process. Finally the final profile and circulation of the merged vortex is determined: the two thirds of the total circulation of the two initial vortices is contained in the newly formed vortex.
\end{abstract}

(c) 2007 Elsevier Masson SAS. All rights reserved.

Keywords: Vortex; Merging; Numerics

\section{Introduction}

The description and understanding of topology changes in velocity fields may be central to study singular behaviours, enstrophy production or dissipation in fluid flows. For truly three-dimensional flows, such processes are within the heart of three-dimensional turbulence or vortex reconnection [1]. In two-dimensional flows, vortex merging [2-8] is the principal ingredient of the inverse cascade [9-11] although three vortex interactions may be also significant. It also explains the thickening of the mixing layer width [12] and is considered in the context of aircraft wakes [13,14]. In meteorology or geophysics, dispersion of passive scalars is partly governed by the merging of large scale vortices. For instance, the plankton may be trapped or untrapped during such events [15]. In such contexts, however, the coalescence of vortices is affected by the Coriolis force [16] which is also the main reason why large geophysical flows are mainly $2 \mathrm{D}$ dynamics. In the present work, our purpose is to perform a quantitative study of merging on the simplest case of two-dimensional interactions i.e. a pair of two-dimensional co-rotating equal vortices. More precisely we identify the mechanisms that occur during this process and analyse their Reynolds number dependence. This is performed using high Reynolds number simulations, a tool which authorises the phenomenon to be studied in a "clean" context. In real experiments, intrusive measurements are present and other effects may be preponderant or else interfere too strongly with the two-dimensional merging itself. For instance, one cannot avoid three-dimensional effects such as finite length

\footnotetext{
* Corresponding author.

E-mail address: josseran@1mm.jussieu.fr (Ch. Josserand).
} 
effects that propagate along vortices [3] or three-dimensional instabilities like the elliptic instability [17,18]. In real experiments, it is difficult to reach because of three-dimensional effects, a "two-dimensional" situation for Reynolds numbers above 1000 [3].

The description of the overall merging process can be done separating four stages to be defined accurately below. Before merging (see Fig. 1(a)), i.e. when vortices are far apart, the vortex pair dynamics may be described by four major features. Firstly, similarly to point vortices, the two-vortices rotate around the total vortex centroid. Secondly, vorticity contours are elliptically deformed by the local strain induced by each vortex on its companion. This is reminiscent of the effect of an external strain on an isolated vortex (see the asymptotic analysis [19,11]) but with the additional feature of a rotating frame [20]. Thirdly, the vorticity field is rearranged by vorticity waves propagating on each vortex [5]. Fourthly, the cores of both vortices increase due to the viscous diffusion. This last effect modifies the ratio between vortex radius and the separation distance between the two vortices in such a way that a critical value is reached at which merging always takes place.

Several works have proposed to understand this critical ratio in terms of the stability of nonlinear finite amplitude states. This idea was applied on vortex patches by following a family of nonlinear states and their stability [21-24] or through numerical simulations [25]. More recently, the same approach has been extended in an inviscid context to various nonuniform vorticity profiles [26,4] or else to uniform vorticity region which is not formed of two disconnected domains i.e. a dumb-bell shape patch [7]. This latter approach recovers the limiting V-states obtained in [27]. The stability analysis of these configurations provides a critical value for the onset of merging. It has been also shown that these equilibria can be understood in terms of a single vortex embedded in an external deformation field due to the companion vortex [28]. Experimentally, a critical value has been found [3,6]. In Section 3, the procedure exploited in these experiments is numerically re-examined showing the bias that it contains.

Once the critical ratio is attained, the merging occurs as a rapid motion: a central zone is created where the major part of the initial vorticity is concentrated while vorticity is also ejected in an outer region in the form of two spiral filaments (see Fig. 1 (b) and (c)). It is known that these latter structures are stabilised by the strain produced by the central zone [29]. At the end of merging, the two vorticity maxima merge in a unique one and a single bigger vortex is formed (see Fig. 1(d)). While the first rotating phase and the onset of merging have been repeatedly analytically and numerically analysed, the merging process itself has not been studied in a precise and quantitative way. In this respect, contour surgery $[23,30]$ or hyperviscosity methods are limited since the effect of Reynolds number cannot be explicitly discussed in this context. In the spirit of the seminal work [31], we have used Navier-Stokes direct numerical simulations at high Reynolds numbers. We raise several questions regarding features that appear during merging. First, we analyse during the merging itself which vorticity zone is preponderant in bringing vortices together. It appears that filaments are not the only part of the flow that contributes to the decrease of the vortex distance. Thereafter, we analyse the mechanism of the plateau (Fig. 2(a)), a stage which was identified in [3,6]. An advection diffusion process is shown to be at work in that phase. Finally we determine once merging is completed, the profile of the central vortex and how much circulation has remained trapped within the unique vortex i.e. has not been lost inside spiral filaments. In a way, this quantity can be interpreted as the percentage of vorticity transferred into larger scales since the merging of equal vortices can be viewed as the simplest physical situation which contains one of the basic mechanism of the inverse cascade. For turbulence, this point may be important for building a simplified model of the process in the spirit of [32]. However this endeavour would necessitate to address the more general merging of two asymmetric vortices.

The paper is organised as follows. In Section 2, dimensionless governing equations are presented and the numerical procedure used to simulate them is given. Some general considerations on the dynamics are also introduced. In Section 3, the computation of the critical size and its origin are discussed. Section 4 analyses the contributions of the various vorticity regions to the vortex motion during the rapid stage of merging. Finally, Section 5 is concerned with the plateau region and is also devoted to an analysis of the final state which is produced once merging is completed.

\section{Numerical implementation}

Let us consider the two-dimensional velocity field $\left(u_{x}(x, y), u_{y}(x, y)\right)$ of an incompressible viscous fluid of kinematic viscosity $v$. The simulation is initiated with two identical vortices $(i=1,2)$ characterised by a Gaussian vorticity profile $\omega_{i}(x, y)$

$$
\omega_{i}(x, y)=\frac{\Gamma}{\pi r_{0}^{2}} \exp \left(-\frac{\left(x-x_{i}\right)^{2}+\left(y-y_{i}\right)^{2}}{r_{0}^{2}}\right), \quad i=1,2 .
$$




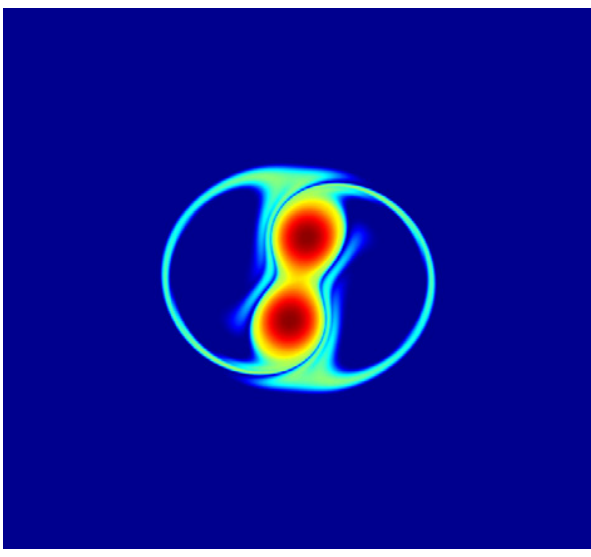

(a)

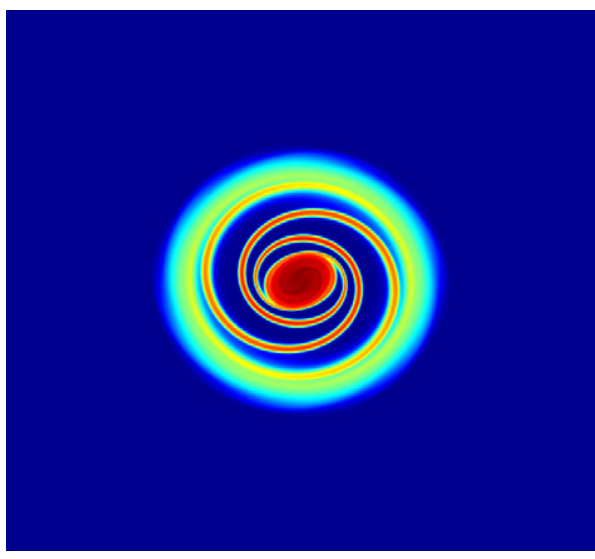

(c)

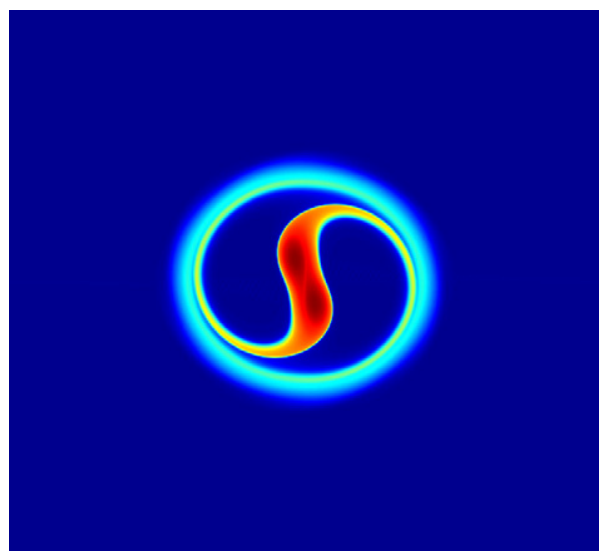

(b)

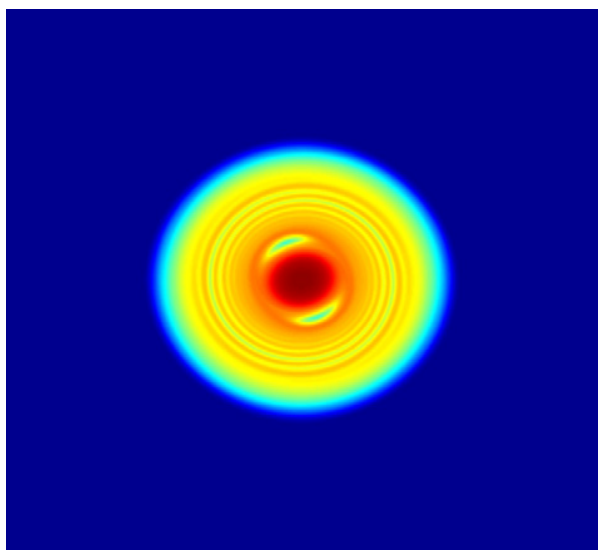

(d)

Fig. 1. Isocontours of the vorticity distribution in a log-plot at four subsequent stages corresponding to $t^{*}=5,36.5,38$ and 46 respectively. This numerical experiment corresponds to a Reynolds number $R e=50000$.

Experimental measurements show that this profile appropriately fits the isolated true vorticity distribution once a vortex has been generated by vorticity layer roll-up. The vortex layer could originate from impulsively rotated flat plates [18] or from the wake behind two wings [6]. The Gaussian profile is also linked to the self-similar solution of the vorticity diffusion equation. More precisely, once the roll-up is completed, the vorticity distribution relaxes towards a quasi-steady state through decaying Kelvin oscillations [5]. This state is a Gaussian vortex deformed by the presence of the companion vortex. In the present work, we do not focus on this equilibration phase and we waited until such a quasi-equilibrium was reached. Practically, we started the simulation with a very small ratio $r_{0} / D_{0}=0.05$ where $D_{0}$ is the initial separation distance between the two vorticity maxima $D_{0} \equiv \sqrt{\left(x_{2}-x_{1}\right)^{2}+\left(y_{2}-y_{1}\right)^{2}}$, and run the numerical simulations at high Reynolds number $R e=\Gamma / v=30000$ until the Kelvin waves were damped. At this time, the vortex was close to the form of Eq. (1) but the effective $r_{0}$ was measured to be $r_{0} / D_{0} \sim 0.1$ (see the discussion on the influence on the vortex shape in [8]). This latest flow was taken to be the true initial conditions for most of our calculations. However for very high Reynolds numbers (above 15000), we used an initial condition with a higher $r_{0}$ (namely $r_{0} / D_{0}=0.18$ ).

Since the two vortices are equal, the flow is completely described by two dimensionless parameters, namely the Reynolds number $R e=\Gamma / v$ and the ratio $r_{0} / D_{0}$. Vortex merger is studied using a simulation of the two-dimensional Navier-Stokes equations in a rectangular box of size $L_{\text {box }}$ which is always chosen to be equal to $2 \pi D_{0}$. The equation for the vorticity field $\omega=(\boldsymbol{\nabla} \times \mathbf{u}) \cdot \mathbf{e}_{z}=\partial_{x} u_{y}-\partial_{y} u_{x}$ writes:

$$
\partial_{t} \omega+\mathbf{u} \cdot \nabla \omega=v \Delta \omega .
$$


When written in terms of a stream function $\psi(x, y)$ and nondimensionalised using length scale $L_{\mathrm{box}} /(2 \pi)$ and time scale $L_{\text {box }}^{2} /\left(4 \pi^{2} \Gamma\right)$, this governing equation reads

$$
\begin{aligned}
\partial_{t}(\Delta \psi)+\mathbf{u} \cdot \nabla(\Delta \psi) & =\frac{1}{\operatorname{Re}} \Delta^{2} \psi \\
\text { with } u_{x}=\frac{\partial \psi}{\partial y}, u_{y} & =-\frac{\partial \psi}{\partial x}, \omega=-\Delta \psi .
\end{aligned}
$$

The computation box is now a square of length $2 \pi$ and periodic boundary conditions are taken:

$$
\psi(x+2 \pi, y)=\psi(x, y+2 \pi)=\psi(x, y) .
$$

In the following, only dimensionless quantities will be considered. In these dimensionless separation distance $L_{0} \equiv$ $2 \pi D_{0} / L_{\text {box }}=1$ and the dimensionless initial core size $a_{0} \equiv 2 \pi r_{0} / L_{\text {box }}$ is equal to 0.1 or else 0.18 . In this periodic numerical box, such values ensure that mirror vortices do not influence the overall process. Note that, because of periodic boundary conditions, the circulation around the box must be zero. A residual constant negative vorticity over the full numerical box must be hence present of circulation $-\Gamma$. This feature however does not perturb the vortex dynamics as shown in the appendix of [34]. In the following, the contribution due to this residual vorticity is always properly subtracted in the physical quantities obtained in our simulations.

The dimensionless Navier-Stokes equations are simulated using a pseudo-spectral Adams-Bashforth algorithm based on two-dimensional fast Fourier transforms, using the efficient library routine FFTW ("Fastest Fourier Transform in the West") [33]. Aliasing is taken into account in a standard manner by decreasing of a factor $3 / 2$ the relevant number of spectral modes.

Various simulations have been performed in which the Reynolds number $R e$ range from $R e=1000-100000$, the number of points $N$ from $N=1024$ to $N=2048$. The time step $\delta t$ varies from $\delta t=5 \times 10^{-4}$ to $\delta t=10^{-3}$ so that the CFL number is below $10^{-2}$ for the initial condition and remains of the order of 0.1 for the highest vorticity gradients regions. It means that we are satisfying the usual CFL condition. The code accuracy has moreover been checked by doubling the number of points.

\section{Premerging period and merging time}

\subsection{The pre-merging period}

Before merging, the center of the vortices drift toward each other (see Fig. 2(a)) and rotate with an almost constant angular velocity $\Omega_{\text {ang }}$. This latter quantity computed using the line joining the two vortex maxima, is practically equal to the value $1 /\left(\pi L_{0}^{2}\right)$ obtained for point vortices [24]. In the corotating frame, the flow is steady up to a standard viscous diffusion effect and to the slight decrease of $L(t)$, the distance between vortex centers: its streamfunction $\Psi=\psi-\left(\Omega_{\mathrm{ang}} / 2\right)\left(x^{2}+y^{2}\right)$ is displayed in Fig. 3. The idea of moving in this rotating frame has been already employed in [34,31] and more recently in [5]. Moreover it was used to compute the onset of merging in [6]. The flow topology and dynamics are characterised by the presence of fixed points: two elliptical points $O_{1}$ and $O_{2}$ associated to vortex centres and three hyperbolic points $H_{0}, H_{1}$ and $H_{2}$. Such points are important for the merging process since they define various separatrix: one heteroclinic orbit that connects $H_{1}$ and $H_{2}$ and an homoclinic orbit that goes through $H_{0}$. Note that in the case of asymmetric vortices, this topological structure is not always valid. However for weak enough asymmetrical vortices, this topology is still pertinent. Reversely, if one vortex is much stronger, its influence can dominate the other one dynamics even in its core. These orbits define four main regions: outside the heteroclinic orbit (I), in between the heteroclinic and homoclinic orbit (II) and inside the homoclinic orbit (III and IV). In this quasi-steady state, the streamfunction does not change much and vorticity is slowly evolving because of viscous diffusion. However these quantities evolve so that, inside the heteroclinic orbit and except close to hyperbolic points, a relationship between streamfunction and vorticity always exists. During this premerging period, the main temporal change is described by the viscous diffusion of each vortex core size. More precisely if $x$ denotes the direction of the line joining the two vortex maxima respectively situated at $x=x_{1}>0$ and $x_{2}=-x_{1}$, a dimensionless core size $a(t)$ can be defined for the vortex located at $x>0$ as

$$
a^{2}(t)=\frac{\int_{0<x} \omega \cdot\left[\left(x-x_{1}\right)^{2}+y^{2}\right] \mathrm{d} x \mathrm{~d} y}{\int_{0<x} \omega \mathrm{d} x \mathrm{~d} y}
$$




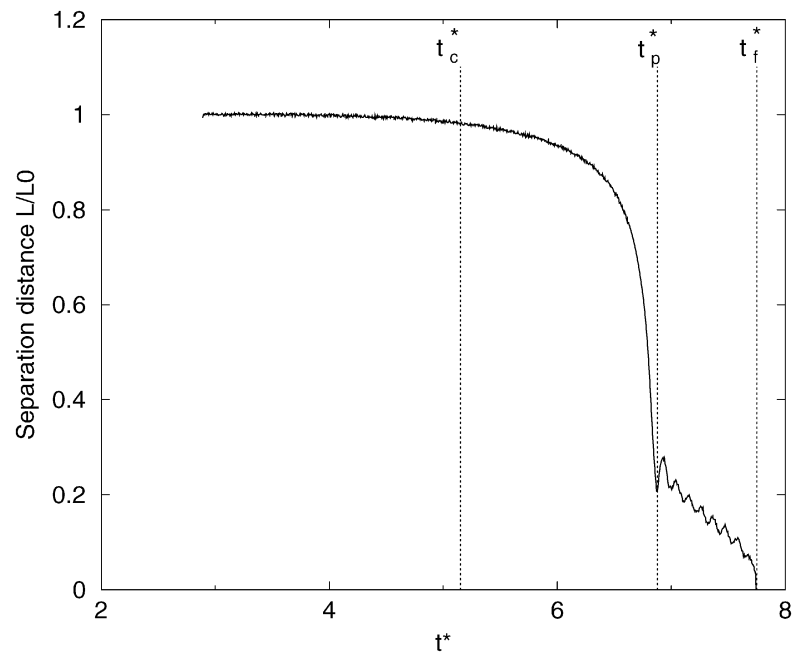

(a)

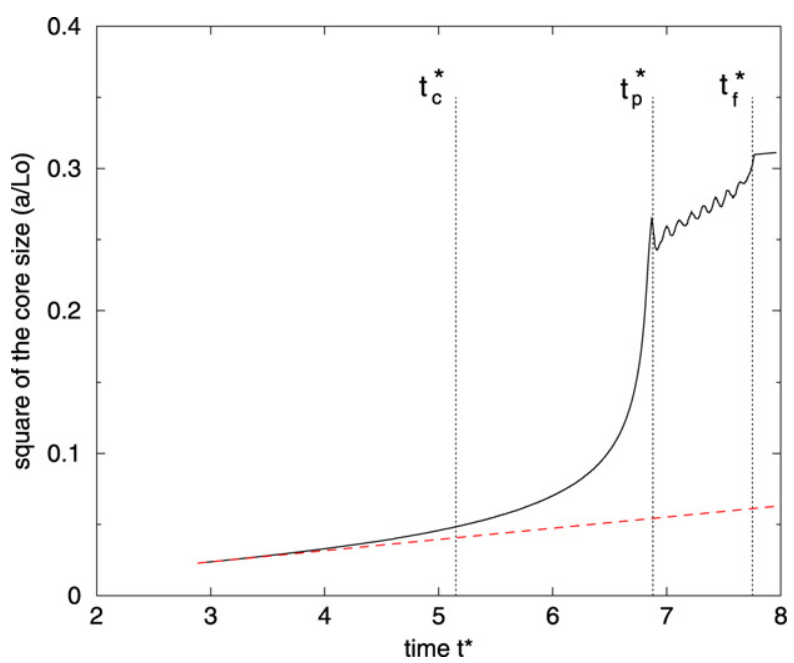

(b)

Fig. 2. (a) Separation distance $L / L_{0}$ between vortex maxima as a function of time $t^{*}$ (numerical experiment at Reynolds number $R e=10000$ ). The four stages (a)-(d) are separated by dashed lines which correspond respectively to $t^{*}=t_{c}^{*}, t^{*}=t_{p}^{*}, t^{*}=t_{f}^{*}$ (see discussion in the text for a definition). (b) Square of the core size $\left(a / L_{0}\right)^{2}$ (see Eq. (4) for the definition of $a$ ) as a function of time $t^{*}$ for $t^{*}<t_{f}^{*}$ for the same simulation. The straight line displays the standard diffusion law $\left(a / L_{0}\right)^{2}=8 \pi^{2} t^{*} / \operatorname{Re}$ (Eq. (5)).

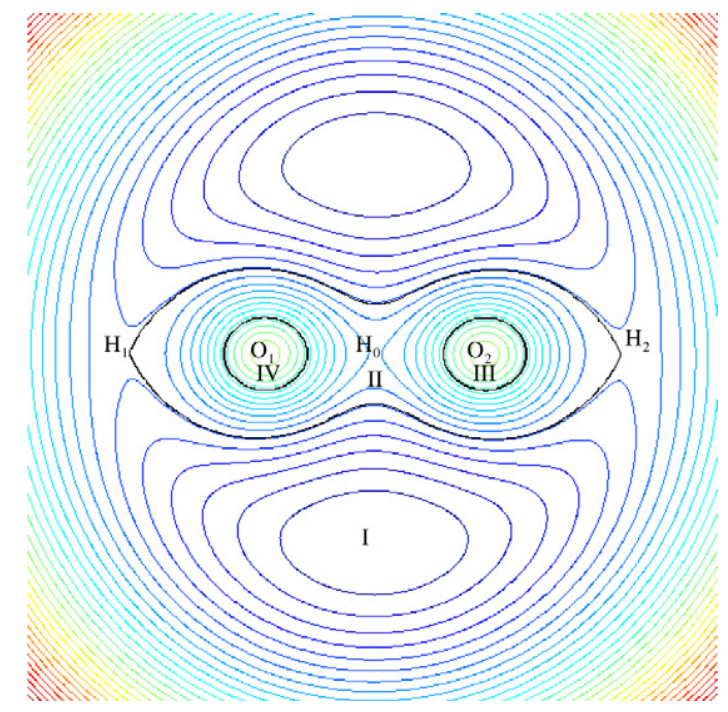

Fig. 3. Streamlines before merging as seen in the co-rotating frame moving with the vortex maxima for $R e=50000$. The two elliptic points $O_{1}$, $\mathrm{O}_{2}$ and three hyperbolic points $\mathrm{H}_{0}, \mathrm{H}_{1}, \mathrm{H}_{2}$ are displayed. The bold streamline, situated inside regions III and IV, delineates a surface of area $\pi a^{2}\left(t_{c}^{*}\right)$ where $a\left(t_{c}^{*}\right)$ denotes the vortex radius (Eq. (4)) at merging onset, the onset being computed via the method based on the existence of a quasi-equilibrium state.

and similarly for the companion vortex with obvious changes. To be rigorous, the centroid of each vortex would be needed instead of the vortex maximum. Before merging it has been checked that this quantity is equal to the vorticity maximum up to mesh size uncertainty. When the initial core size $a_{0}$ is small with respect to $L_{0}$, the evolution of $a(t)$ is truly similar to that of an isolated Gaussian vortex: the core size is increasing as $a^{2}=a_{0}^{2}+4 t / R e$. If $t_{0}$ denotes the time at which the vorticity support was reduced to a point, the previous relation may be rewritten as

$$
\left(\frac{a}{L_{0}}\right)^{2}=\frac{8 \pi^{2}}{\operatorname{Re}} t^{*} \quad \text { with } t^{*} \equiv \frac{t-t_{0}}{2 \pi^{2} L_{0}^{2}} \text { and } t_{0} \equiv-\operatorname{Re} a_{0}^{2} / 4
$$




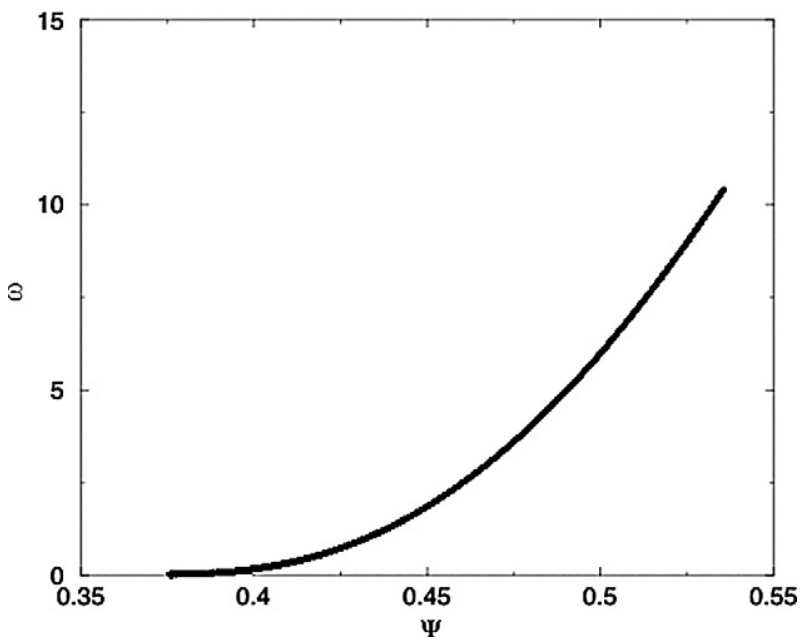

(a)

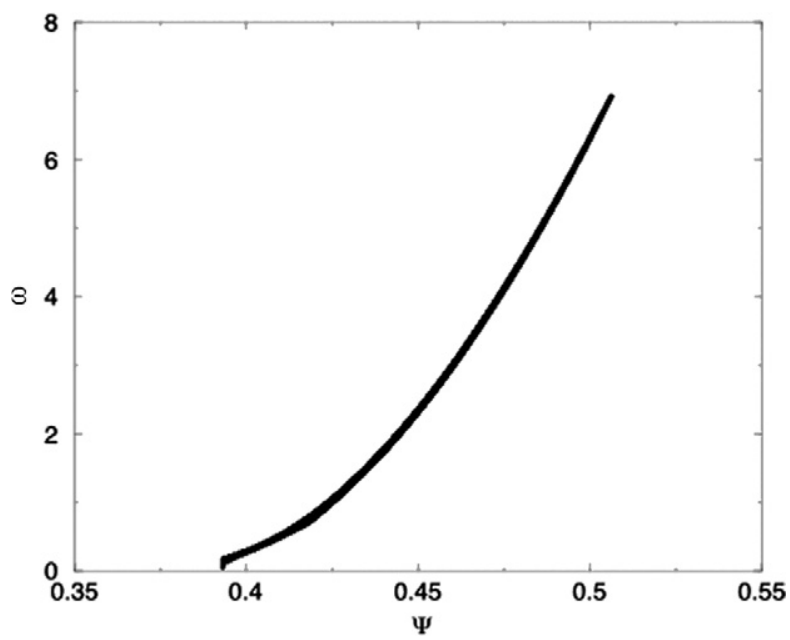

(b)

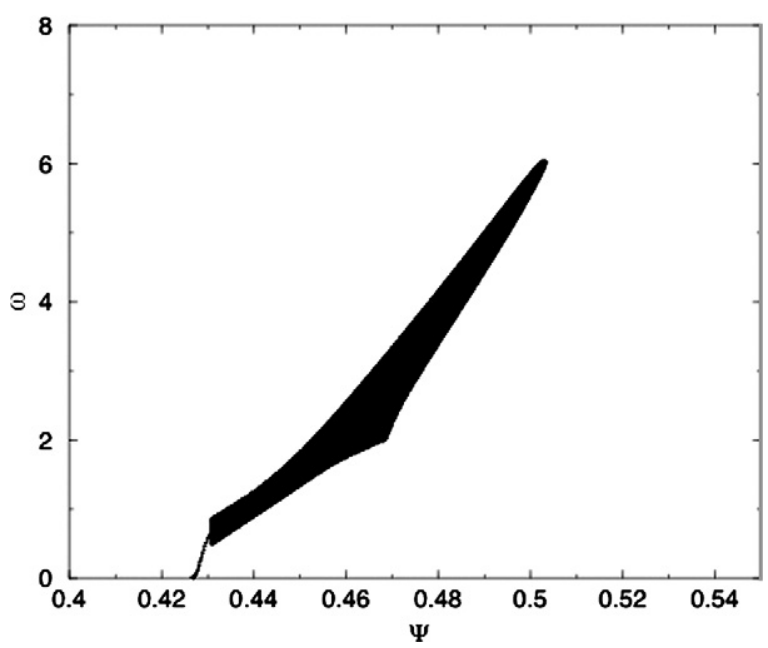

(c)

Fig. 4. Scatter plot $(\Psi, \omega)$ for three different times (a) during stage (a), (b) just before merging onset, (c) after merging onset it i.e. during stage (b) The merging onset is computed using the method based on the quasi-equilibrium hypothesis.

where $t^{*}$ stands for the time delay from $t_{0} \equiv-\operatorname{Re} a_{0}^{2} / 4$ computed with respect to the turnover period $2 \pi^{2} L_{0}^{2}$ of a point vortex pair of identical circulation-here equal to one - and of separation distance $L_{0}$. This latter equation can be interpreted as a relation between time $t^{*}$ and ratio $a / L_{0}$. Evolution (5) however does not persist during the whole premerging period in which the quasi-equilibrium is still present: Eq. (5) clearly breaks down (see Fig. 2(b)) when approaching the second stage. Concomitantly the separation distance $L(t) / L_{0}$ between the two vortex centres does not remain constant during the whole premerging period (see Fig. 2(a)). Moreover this latter quantity does not show any sharp transition from stage (a) to (b): it is hence difficult to determine from such a unique measurement the onset of merging.

During the premerging period, one assumes that the solution corresponds to a quasi-equilibrium in the co-rotating frame. This implies that, at leading order, there exists a functional relationship between vorticity $\omega$ and streamfunction $\Psi$ [5]. This assumption is justified by the scatter plot taken at a time taken before merging (see Fig. 4(a)) where a one-to-one character is clear. After merging onset (see Fig. 4(c)), the scatter plot has diffused: during that period, the dynamics is inviscid but does not correspond to an equilibrium. Near merging onset (see Fig. 4(b)), one can observed the qualitative change of the one-to-one character of the relation for the low values of $\Psi$ and $\omega$. This feature, more than the deviation from Eq. (5) may be used to pinpoint numerically the merging onset (see below). 


\subsection{The merging onset}

The onset of merging, in particular the definition of a merging time $t_{c}^{*}$ and critical ratio $(a / L)_{c}$, was determined in $[3,6]$ using data for the separation distance $L(t) / L_{0}$ collected in experiments carried out at different Reynolds numbers. In the present work, the adequacy of this experimental procedure is analysed using numerical simulations performed at Reynolds numbers ranging from $R e=1000$ to $R e=50000$. The dependence of the merging time and critical ratio thus computed is examined with respect to the Reynolds number on a large interval of Reynolds numbers which cannot be reached via experimental situations because it is difficult to avoid experimentally the presence of three-dimensional elliptical instabilities.

The trick used in [3] can be explained as follows. Let us assume that merging is essentially an inviscid dynamics that takes place once a critical value $(a / L)_{c}$-independent of Reynolds number-of the ratio $a / L$ is reached. The overall process is then splitted into two consecutive phases: a diffusive phase before merging onset and a purely convective phase after onset. During the diffusive phase, one assumes that Eq. (5) is valid and that $\left(L / L_{0}\right)=1$ which implies that weak filaments ejected in the outer region and advected along the external heteroclinic orbits, are not taken into consideration. Both these constraints however were shown above not to be correct before the merging. During the convective phase, the two vortex centres rapidly move nearby and Eq. (5) breaks down. The existence of two different evolutions implies that $t_{c}^{*}$ be proportional to the Reynolds number

$$
t_{c}^{*}=A \operatorname{Re} \quad \text { with } A=\frac{1}{8 \pi^{2}}\left(\frac{a_{c}}{L_{0}}\right)^{2}
$$

and that the decrease of the separation distance $L(t)$ during the convective stage is independent of the Reynolds number. In particular the time interval $B_{q}$ after merging onset which is necessary for the vortex distance to be reduced to a percentage $q$ must be Reynolds number independent. As a consequence, the overall time $t_{q}^{*}$ necessary to reach this distance, i.e. $L\left(t_{q}^{*}\right)=q L_{0}$ depends only on $\operatorname{Re}$ as

$$
t_{q}^{*}=A R e+B_{q}
$$

with a coefficient $A$ a priori independent of $r$. The slope $A$ is computed from a least-squares fit of the data set. From this value of $A$, the critical time $t_{c}^{*}=A R e$ can be also computed. In Fig. 5(a), the time $t_{q}^{*}$ is shown as a function of Reynolds number for $q=50 \%$ : its variation is indeed linear with Reynolds number for the considered interval in agreement with the above scenario. A small kink however can be observed at relatively small Reynolds number $R e \leqslant 5000$. For such Reynolds numbers, the time scales of diffusion and convection are not so well separated which may cause a bias in the linear relation between $t_{q}^{*}$ and $R e$. In addition, the coefficient $A$ is almost constant with respect to $q$ (see Fig. 5(b)) and equal to $A=5.8 \times 10^{-4}$. The values above $q=0.8 \operatorname{did}$ not provide reliable values for $A$ because of the change in $L$ before merging onset. Similarly, below $q=0.3$ another problem arises that is discussed in Section 5. The critical value $(a / L)_{c}$ is then $(a / L)_{c}=\sqrt{8 \pi^{2} A} \sim 0.214$. This value does not compare satisfactorily to the experimental result $0.24 \pm 0.1$ found in [4] using this very same procedure at smaller Reynolds number. This discrepancy may be attributed to the relatively low Reynold number used in experiments. However the value $(a / L)$ computed numerically at critical time $t_{c}^{*}=A R e$ for each Reynolds number curves should not depart too much from the value $(a / L)_{c}=0.214$. The curve of Fig. 6 indicates that this is not the case which contradicts the main hypothesis! This result is to be related to the weak validity of Eq. (5) until merging. The above method though helpful, leads to inconsistent results when completely analysed.

The previous criterion was essentially based on Eq. (5), which refers to the diffusion process for a single vortex. It would be more satisfactory to build this criterion on the existence of a dynamical equilibrium of the vortex pair in the inviscid context. That is why we propose below to define a functional of vorticity which is based on the existence of such an equilibrium. For the viscous case, such a procedure imposes much less constraints on the fluid motion: one simply assumes the existence of a viscous quasi-equilibrium. At leading order, this viscous quasi-equilibrium reads

$$
\omega=\omega_{0}(r, \theta-\Omega(\lambda) t, \lambda)+\frac{1}{R e} \omega_{1}
$$

where $\lambda$ is a continuous parameter that defines a family of inviscid equilibria $\omega_{0}$ rotating with angular velocity $\Omega(\lambda)$. For instance $\lambda$ can be chosen to be equal $(a / L)^{2}$. The shape of these equilibria looks like Fig. 1(a) with a vortex ring surrounding the two vortices. It is also reminiscent of the model solution devised in [7]. Implementing the above 


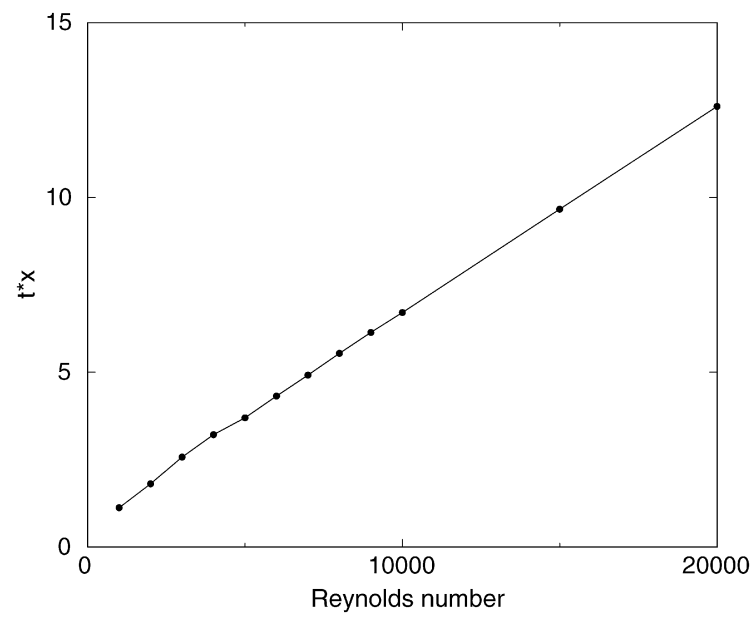

(a)

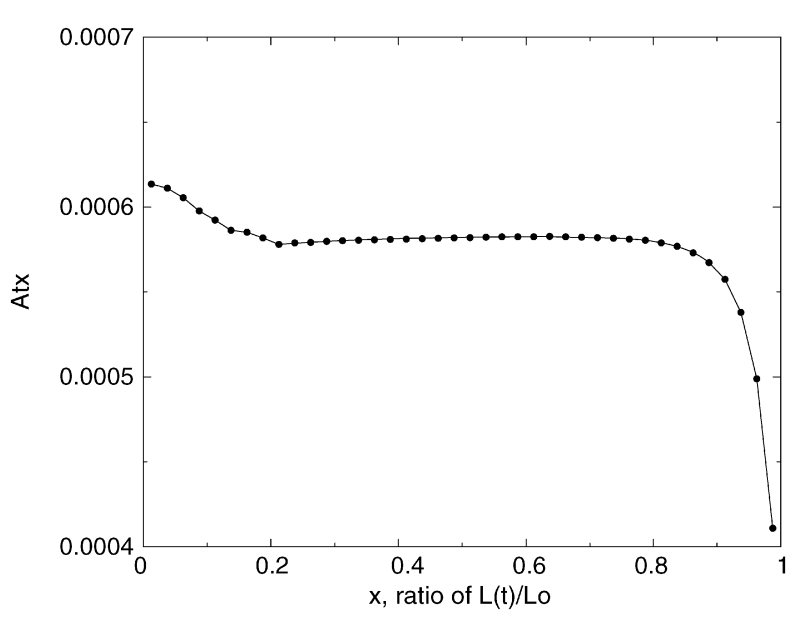

(b)

Fig. 5. (a) Time $t_{q}^{*}$ as function of Reynolds number $R e$ for $1000<R e<20000$ and $q=L\left(t_{q}^{*}\right) / L_{0}=0.5$. (b) Values of $A$ as a function of various fractions $q$. For the interval $0.3<q<0.8$, the different values are well approximated by the constant $A=0.00058$ which has been chosen as the value at $q=0.5$.

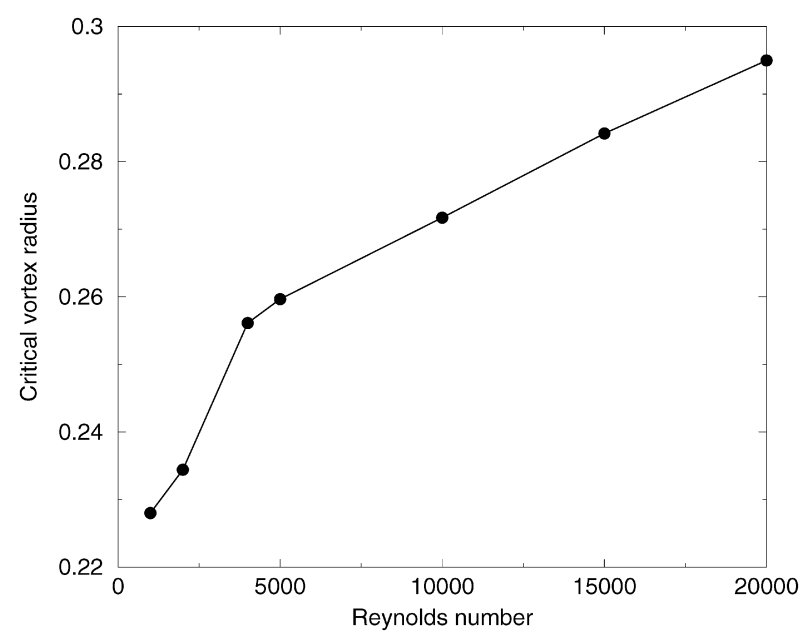

Fig. 6. The critical ratio $(a / L)_{c}$ as a function of the Reynolds number $R e$, to be compared to the value 0.24 found in the experiment [4].

ansatz in the vorticity equation, it is readily seen that in the co-rotating frame, the term $\mathbf{u} \cdot \nabla \omega$ scales in Reynolds number like the viscous term $\Delta \omega / R e$. We introduce thus the vorticity functional $F$ defined as:

$$
F=\frac{I}{J} \quad \text { with } I(t)=\int[\mathbf{u} \cdot \nabla \omega]_{\text {corot }}^{2} \mathrm{~d} x \mathrm{~d} \text { and } J(t)=\frac{1}{R e^{2}} \int[\Delta \omega]^{2} \mathrm{~d} x \mathrm{~d} y
$$

where the velocity $u$ is computed in the co-rotating frame with angular velocity associated with the vorticity maxima

$$
[\mathbf{u} \cdot \nabla \omega]_{\mathrm{corot}} \equiv[\mathbf{u} \cdot \nabla \omega]-\Omega\left(-y \frac{\partial \omega}{\partial x}+x \frac{\partial \omega}{\partial y}\right) .
$$

The inviscid equilibrium assumption implies that, before merging, the function $F$ depends on the ratio $a / L$ only, independent of Reynolds number and is almost equal to 1 . When the quasi-equilibrium assumption is no more valid, function $F$ should change with Reynolds number and rapidly departs from unity. Fig. 7 shows the function $F$ for different Reynolds numbers as a function of $a / L$. The expected behaviour is observed with a strong departure from unity when $a / L$ increases and reach values above 0.25 . It is thus tempting to use this functional $F$ to define the merging onset. However, the exponential-like growth of the function $F$ does not allow for a clearcut definition of the 


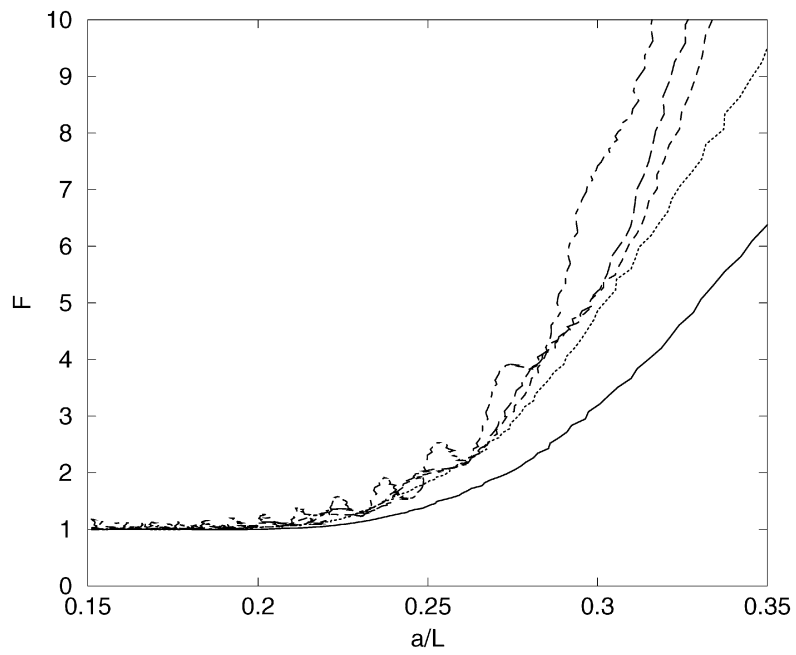

Fig. 7. Value of $F$ (see Eq. (9)) as a function of $a / L(t)$ for Reynolds numbers $R e=1000,2000,4000,5000$ and 10000 (the higher the curve, the higher the Reynolds number). Notice that the curve for $R e=10000$ shows numerical oscillations which can be attributed to the Cartesian structure of the mesh function amplified by the $\mathfrak{R}^{2}$ factor in the definition of $F$.

merging. In the present work, we ask that this modification should not be larger than ten percent: one obtains thus a critical size equal to $a_{c} / L=0.22$ which, by definition, is independent on the Reynolds number. Consequently, the critical time $t_{c}^{*}$ no longer exhibits a linear dependence on the Reynolds number. Note however that this criterion which is based on a more satisfactory viewpoint i.e. inviscid dynamical properties of the flow, does not show as it stands a remarkable improvement. The idea of a quasi-equilibrium needs to be studied further on.

\section{Second stage: A rapid decrease}

When merging $t^{*}>t_{c}^{*}$ starts the dynamics is quite different. As seen on Fig. 2, the separation rapidly decreases for $t_{c}^{*}<t^{*}<t_{p}^{*}$. This stage is followed for $t_{p}^{*}<t^{*}<t_{f}^{*}$ by a plateau until the two vorticity maxima merge. In this section, we analyse the time evolution between $t_{c}^{*}$ and $t_{p}^{*}$. Several notions that appear to be useful before merging (corotating frame, regions inside homoclinic or heteroclinic orbits), may be generalised for this inviscid second stage. The angular velocity of the co-rotating frame is chosen in a different way after merging onset. In the previous section, i.e. before onset, it was based on the rotation of the line intersecting the two vorticity maxima. For a quasi-steady state, this procedure is valid since the entire flow is co-moving. When the flow departs from this quasi-equilibrium, one is bound to find a more global definition based on the characteristics of the whole flow such as moments of the vorticity distribution. One may require the outer potential field to be as steady as possible. This potential field can be written as an expansion in terms of vorticity moments. The leading contribution in this expansion is related to the equivalent axisymmetric vortex and does not change during merging since the total circulation $\Gamma$ is conserved and the global centroid remains at the initial rotation centre. The following term in this expansion is given by an elliptic contribution which depends on two multipole coefficients

$$
I_{1,2} \equiv \int \omega(x, y)\left(x^{2}-y^{2}\right) \mathrm{d} x \mathrm{~d} y, \quad I_{2,2} \equiv 2 \int \omega(x, y) x y \mathrm{~d} x \mathrm{~d} y .
$$

The axes of the elliptic contribution remain identical if quantity $I_{1,2} / I_{2,2}$ (or its inverse $I_{2,2} / I_{1,2}$ if $I_{2,2}$ is zero initially) is kept constant by an adequate rotation of the reference frame (numerically the angle of this rotation can be easily computed and is displayed on Fig. 8 (bold line). Such a method based on the elliptic contribution clearly differs from the usual method based on vorticity maxima, at the end of the second stage (dashed line on Fig. 8). During this nonequilibrium stage, the method based on the elliptic moment is appropriate since (a) the angular velocity $\Omega_{\text {ellip }}$ based on the "elliptic moment" angle appears to be constant during this second stage, and (b) it takes into account not only the vortex centres but the whole field. Vorticity contours and streamlines computed in the comoving frame based on the elliptic moment are respectively displayed in Figs. 9(a) and 9(b). 


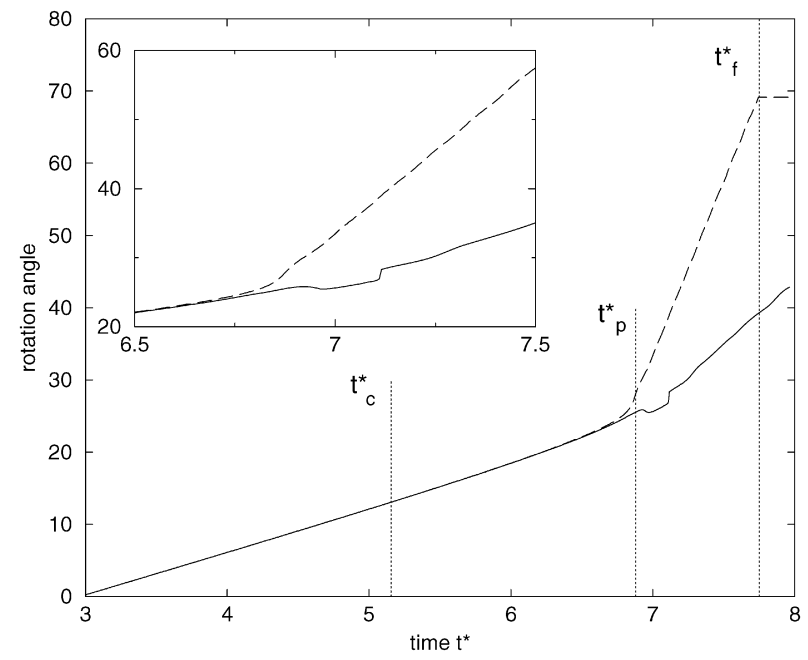

Fig. 8. The rotation angle of the comoving frame based on the elliptic moment (bold line) or on the line joining the vortex maxima (dashed line) as a function of time at $\operatorname{Re}=10000$. Times $t^{*}=t_{c}^{*}=5.15, t^{*}=t_{p}^{*}=6.88$ and $t^{*}=t_{f}^{*}=7.75$ are shown by dotted vertical lines. We observe a kink on the elliptic moment evolution around $t_{p}^{*}$ that we interpret as the ejection of vorticity outside the vortex region.

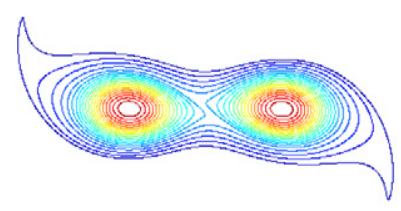

(a)

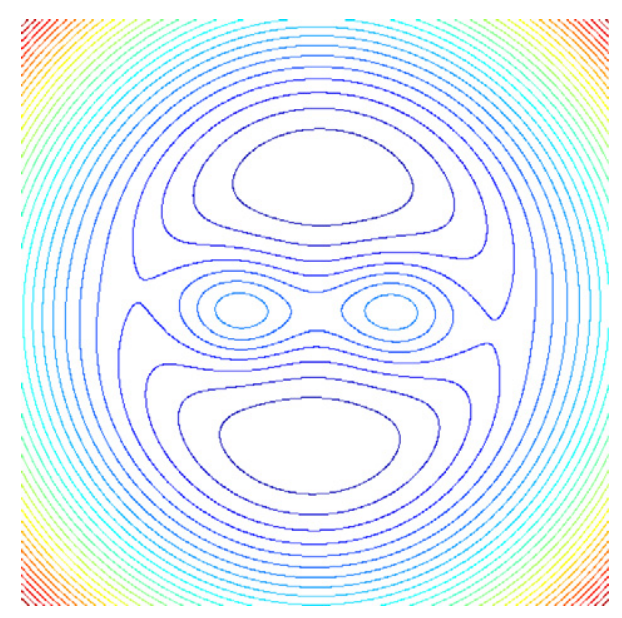

(b)

Fig. 9. Simulation at $R e=10000$. (a) Vorticity isocontours for $t_{c}^{*}<t^{*}<t_{p}^{*}$. (b) At same time $t^{*}$, streamlines of streamfunction $\chi_{\text {ellip }}=$ $\psi-\left(\Omega_{\text {ellip }} / 2\right)\left(x^{2}+y^{2}\right)$ computed in the comoving frame based on the elliptic moment.

Before merging, regions were defined in terms of streamlines. This procedure was pertinent in terms of vorticity dynamics since an one-to-one relation between streamfunction and vorticity existed in that case. When merging starts, this relation is no more valid as seen in the scatter plot of Fig. 4(c). Nevertheless, an outer region I may be still defined based on the heteroclinic orbit of the streamfunction computed in the elliptic co-moving frame $\chi_{\text {ellip }}$ defined by:

$$
\chi_{\text {ellip }}=\psi-\left(\Omega_{\text {ellip }} / 2\right)\left(x^{2}+y^{2}\right) .
$$

The definition of the filament region I is objective and coherent since the streamfunction $\chi_{\text {ellip }}$ is determined via the "steadiness" of the outer potential field. Indeed it has been checked that this heteroclinic orbit is slightly changing between $t_{c}^{*}$ and $t_{p}^{*}$. Inside the heteroclinic orbit, the situation is unsteady and streamlines are no more adequate to describe the dynamics. However, one may still separate regions II, III and IV as follows. During the time interval $t_{c}^{*}<t^{*}<t_{p}^{*}$, vorticity contours surround only one or else two vorticity maxima (see Fig. 9(a)). Regions III and IV are then defined as the regions of maximum area in which vorticity contours are closed connected curved surrounding a unique vorticity maximum during the complete evolution between $t_{c}^{*}$ and $t_{p}^{*}$. Practically, the isovorticity contour 
which goes through the centre of symmetry at time $t_{p}^{*}$, defines a separatrix, reminiscent of the previous homoclinic orbit, which delineates the two vortices at time $t_{p}^{*}$. A percolation algorithm has been used to numerically determine these two zones. Within a large Reynolds number approximation, the vortex boundaries are convected from $t=t_{c}^{*}$ to $t=t_{p}^{*}$ by the flow. At any time $t_{c}^{*}<t^{*}<t_{p}^{*}$, one may thus define the two regions III and IV by convecting the boundaries at $t=t_{p}^{*}$ back in time. Such a procedure determines two vorticity regions which are closed and keep their coherence during the second phase. The area excluding I, III and IV plays the role of region II.

Let us now extend the moment model approach developed by [31] for the two regions III or IV. It is reminded that the moment model was applied on an elliptical vortex patch using an integral form of the Navier-Stokes equation. Let us consider a function $G(x, y)$ of Lagrangian variables and defined on a Lagrangian surface $S$, the Navier-Stokes equation written in the rotating frame of constant angular velocity $\Omega_{\text {ellip }}$, reads in integral form

$$
\frac{\partial}{\partial t} \int_{S} G(x, y) \omega \mathrm{d} x \mathrm{~d} y=\int_{S} \omega\left[\frac{\partial G}{\partial x} \frac{\partial \chi_{\text {ellip }}}{\partial y}-\frac{\partial G}{\partial y} \frac{\partial \chi_{\text {ellip }}}{\partial x}\right] \mathrm{d} x \mathrm{~d} y+\frac{1}{R e} \int_{S} G \Delta \omega \mathrm{d} x \mathrm{~d} y .
$$

Since one assumes this phase to be essentially inviscid, the contribution of the second integral may be neglected so that

$$
\frac{\partial}{\partial t} \int_{S} G(x, y) \omega(x, y) \mathrm{d} x \mathrm{~d} y=\int_{S} \omega(x, y)\left[\frac{\partial G}{\partial x} \frac{\partial \chi_{\text {ellip }}}{\partial y}-\frac{\partial G}{\partial y} \frac{\partial \chi_{\text {ellip }}}{\partial x}\right] \mathrm{d} x \mathrm{~d} y .
$$

By applying the above identity with functions $G(x, y)=1, G(x, y)=x, G(x, y)=y$, the motion $\left(\mathrm{d} X_{i} / \mathrm{d} t, \mathrm{~d} Y_{i} / \mathrm{d} t\right)$ of the vorticity centroids

$$
\begin{aligned}
X_{i} & =\int_{i} x \omega(x, y) \mathrm{d} x \mathrm{~d} y / \int_{i} \omega(x, y) \mathrm{d} x \mathrm{~d} y, \\
Y_{i} & =\int_{i} y \omega(x, y) \mathrm{d} x \mathrm{~d} y / \int_{i} \omega(x, y) \mathrm{d} x \mathrm{~d} y
\end{aligned}
$$

for the two regions $i=\mathrm{III}, \mathrm{IV}$, is obtained as a function of an integral depending on the streamfunction $\chi_{\text {ellip. }}$. Since we are able to quantitatively separate the effect of each vorticity zone on the value of the streamfunction $\chi_{\text {ellip }}$, the role of vortex III, region II and filaments I upon the motion of vortex IV can be completely evaluated. This procedure is thus more general than those previously used. In [31], each vortex dynamics depends on the other vortex regions while the intermediate region II and the filaments region I are not even modelled. On the contrary, experimental works $[3,6]$ put the emphasis as a main mechanism of merging on filaments to create an asymmetry. In these latter works, filament contribution is evaluated by two different procedures: a first one uses an ad hoc symmetrisation of the global velocity field [6], a second one subtracts an equivalent Gaussian vortex [3]. Both approaches define geometrically the filaments. In the present work, these regions are defined in a dynamical way.

On Fig. 10, the contribution to the velocity of a vortex centroid (here vortex IV) is displayed. To be precise only the velocity component along the line joining this centroid to the centre of symmetry is shown. This figure confirms the significance of all zones to the vortex motion. Filaments do play a role but region II is as important. Actually the relevance of region II implicitly appears in [7] in which the family of nonlinear solutions is extended to dumbbell forms. Finally, the other vortex does not contribute significantly except near $t_{p}^{*}$. Indeed, the main effect of this companion vortex consists in the overall rotation which is already accounted by the rotating frame.

Let us examine how streamfunction $\chi_{\text {ellip }}$ is modified during time evolution. Fig. 11 shows its values along the line joining the two vorticity maxima. Before $t_{p}^{*}$, two streamfunction maxima are present which disappear precisely at $t=t_{p}^{*}$ while the vorticity maxima are still present. A simultaneous evolution can be viewed through the Fourier components of the streamfunction

$$
\chi_{\text {ellip }}(r, \theta)=\Psi_{0}(r)+\Psi_{2}(r) \cos (2 \theta)+\Psi_{4}(r) \cos (4 \theta)+\cdots .
$$

Fig. 12 indicates that the axisymmetric component $\Psi_{0}(r)$ becomes the leading component as time proceeds. 


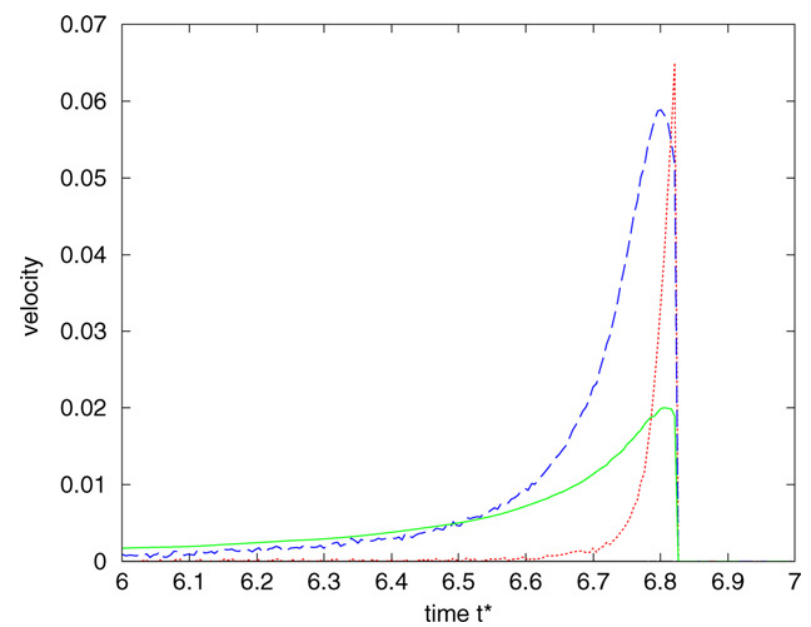

Fig. 10. Contributions of the various zones to the velocity of the vortex IV centroid as a function of time for $t^{*}<t_{p}^{*}$. More precisely, the contributions to the velocity component along the line joining the centroid to the centre of symmetry is shown. Filament region I (bold line), intermediate region II (long-dashed), companion vortex III (dots). Numerical experiment performed at $R e=10000$.

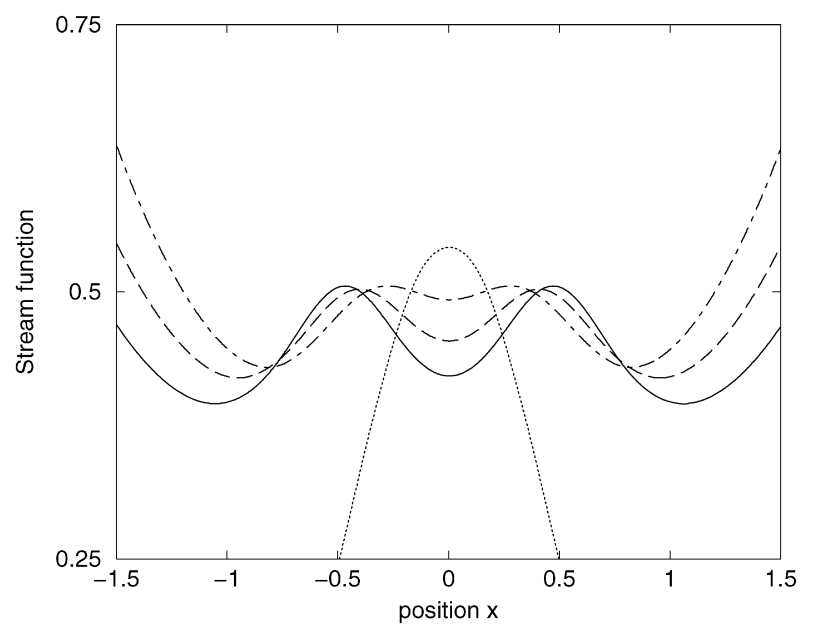

(a)

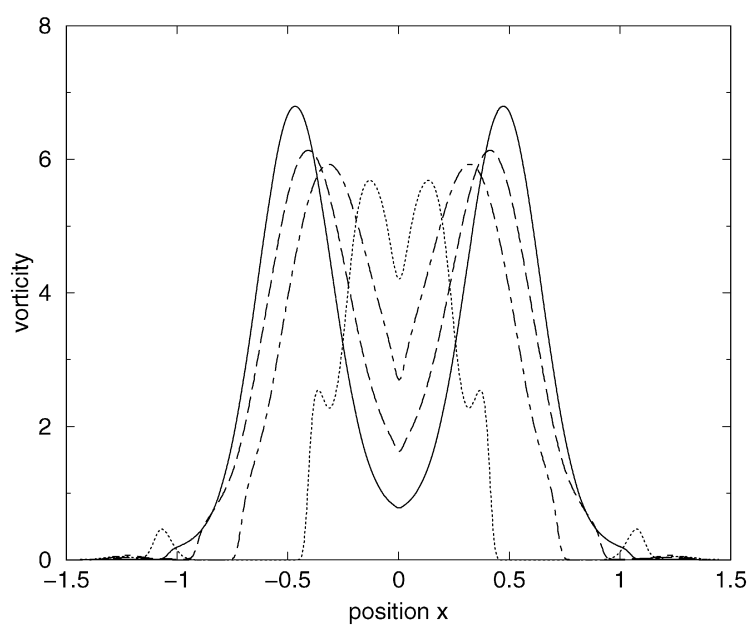

(b)

Fig. 11. Streamfunction (figure (a)) in the rotating frame based on the elliptic moments, as well as vorticity (figure (b)) computed along the line joining the two vorticity maxima at successive times $t_{1}^{*}=t_{c}^{*}$ (continuous line), $<t_{2}^{*}$ (dashed line) $<t_{3}^{*}\left(\right.$ dot-dashed) $<t_{p}^{*}<t_{4}^{*}\left(\right.$ dotted line) $<t_{f}^{*}$. Note that $t_{3}^{*}$ and $t_{4}^{*}$ are close to $t_{p}^{*}$. Numerical simulation performed at $R e=10000$.

\section{The Plateau and the decaying final stage}

In the above section, it was shown that the streamfunction $\chi_{\text {ellip }}$ is mainly axisymmetric after $t_{p}^{*}$. The vorticity dynamics is then close to an advection diffusion process with an axisymmetric convection rather than a pure diffusion process as described in [6]. This streamfunction generates a radial gradient of angular velocity

$$
V_{\mathrm{rg}} \equiv \frac{\mathrm{d}}{\mathrm{d} r}\left[U_{\theta} / r\right] \quad \text { with } U_{\theta}=-\frac{\mathrm{d} \Psi_{0}}{\mathrm{~d} r} .
$$

The deviation of the vorticity field from its axisymmetric component $\left(\omega-\omega_{n=0}\right)(r, \theta)$ (see Fig. 13), thus evolves similarly to a passive scalar advected by an axisymmetric field $\Psi_{0}(r)$ [35,36]. Indeed, the cooperative action of the differential rotation and viscous diffusion is at the origin of an accelerated decay for $\left(\omega-\omega_{n=0}\right)$. For high Reynolds numbers, this action generally brings a multiplicative factor $\exp \left(-n^{2}\left(V_{\mathrm{rg}}\right)^{2} t^{3} / 3 R e\right)$ for all azimuthal modes $n \neq 0$ and thus introduces an accelerated diffusion in $R e^{1 / 3}$ [35]. However near the point for which the differential rota- 


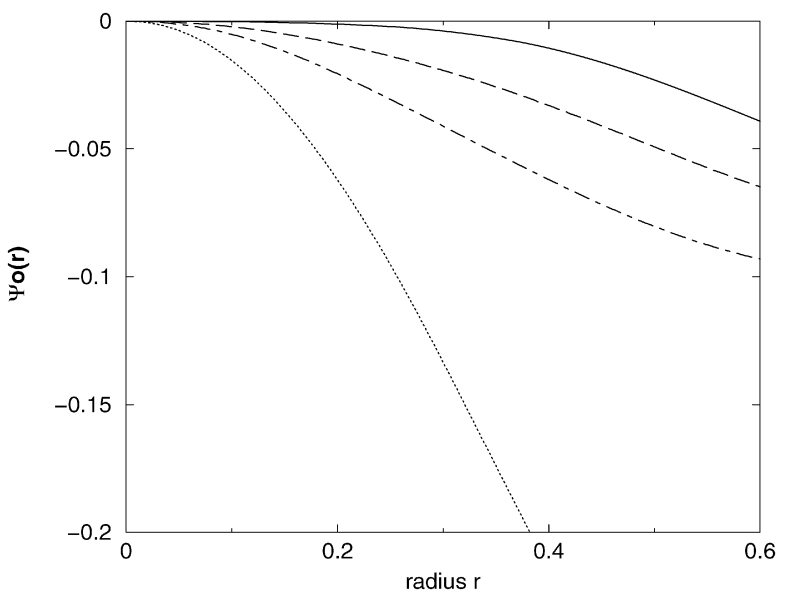

(a)

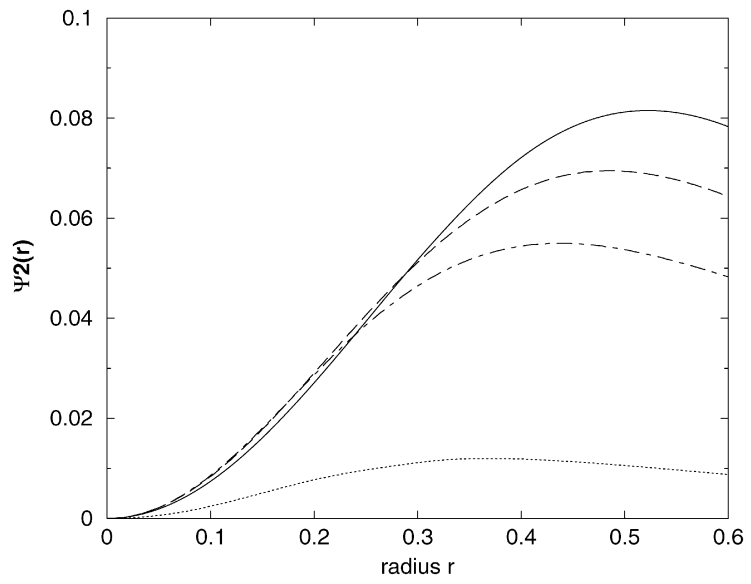

(b)

Fig. 12. (a) Streamfunction Fourier components $\Psi_{0}(r)$. (b) Streamfunction Fourier component $\Psi_{2}(r)$ at $\operatorname{Re}=10000$ for times $t_{i}^{*}(i=1,2,3,4)$ of Fig. 11. Notations of Fig. 11 are used for the corresponding curves.

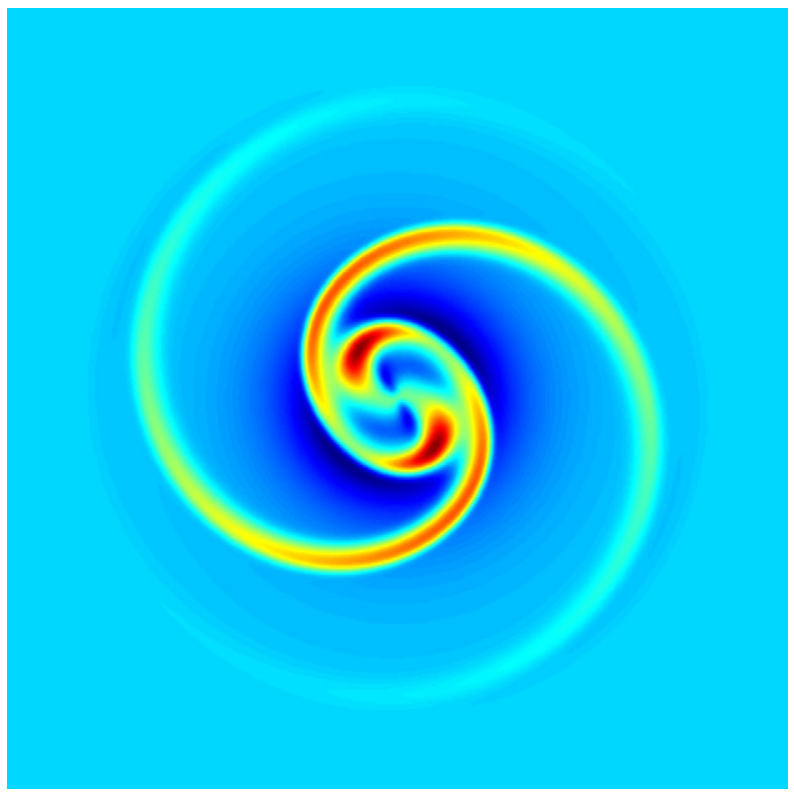

Fig. 13. Contours of nonaxisymmetric vorticity perturbation $\left(\omega-\omega_{n=0}\right)$ where $\omega_{n=0}$ is the axisymmetric vorticity component. Times $t_{p}^{*}<t_{5}^{*}<t_{f}^{*}$ and simulations at $R e=10000$.

tion vanishes for instance at the vortex centre, the asymptotic computations should be modified [36] and provide a scaling $R e^{1 / 2}$ in Reynolds number which is still shorter than the standard diffusion in $R e$. This scenario is confirmed on Fig. 14(a) where it is shown that the longer the plateau size, the larger the Reynolds number and especially on Fig. 14(b) where the duration of the plateau regime is displayed as a function of Reynolds numbers (ranging from 1000 to 100000 ). The line indicating the $R e^{1 / 2}$ scaling fits very well the observed data.

At $t^{*}=t_{f}^{*}$, the two vorticity maxima merge (Fig. 2) and the fusion is complete: the vorticity profile $\omega(r, \theta)$ can be described as a central vortex surrounded by filamentary structures (see Fig. 15(a)). These filaments are wrapped up by differential rotation induced by the closed vorticity region in a way similar to the one studied in [35]. The axisymmetric component $\omega_{0}(r)$ at $t_{p}^{*}$ is shown for various Reynolds numbers Re on Fig. 15(b). In the filament region, these profiles collapse into a single curve. Moreover, the total circulation lost into the filaments has been computed: it is equals to $1 / 3 \pm 5 \%$ of the total circulation of the two initial vortices. Up to numerical errors, this value can be 


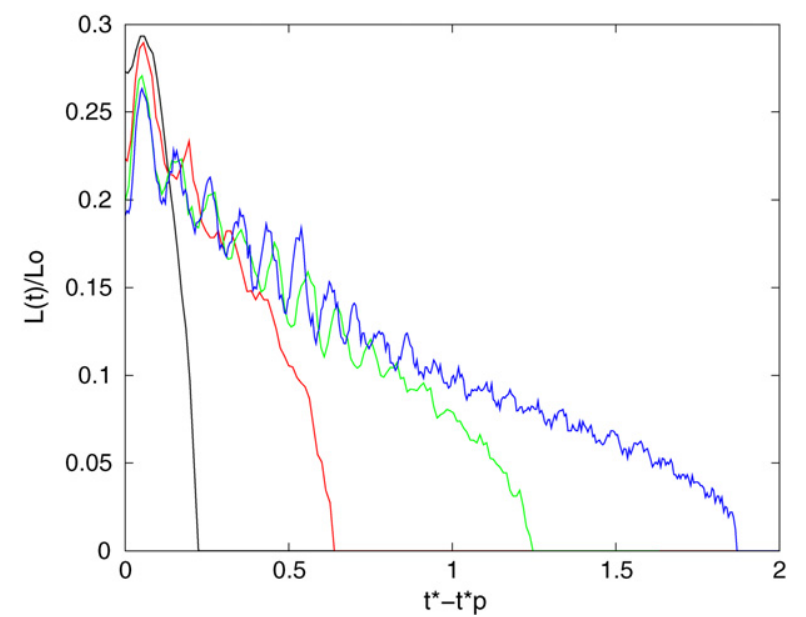

(a)

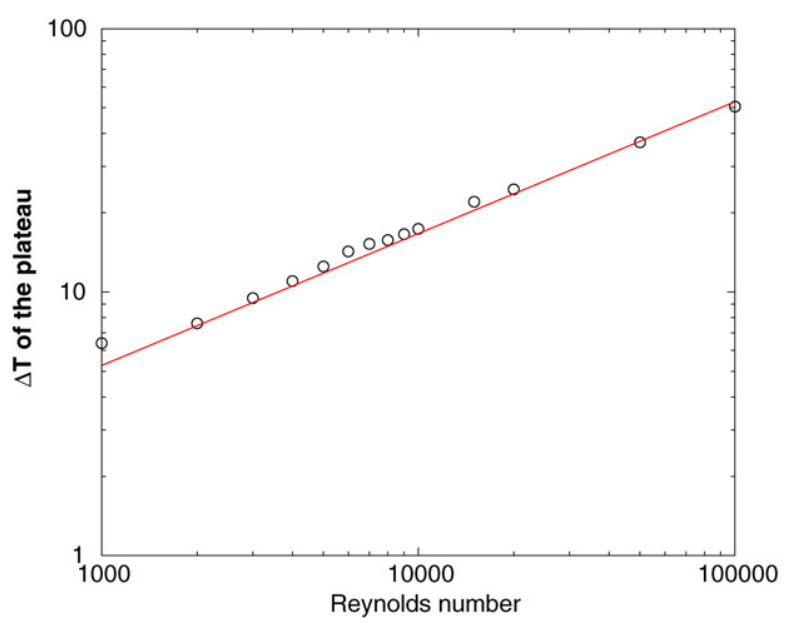

(b)

Fig. 14. (a) Zoom on the plateau regime. The vortex distance $L(t) / L_{0}$ is shown as a function of time $t^{*}$ for $t>t_{p}^{*}$ and for various Reynolds numbers ( $R e=1000, R e=5000, R e=20000, R e=50000$ ). The higher the Reynolds number, the longer the curve. (b) Duration of the plateau region as a function of Reynolds numbers. The straight line corresponds to a $R e^{1 / 2}$ scaling.

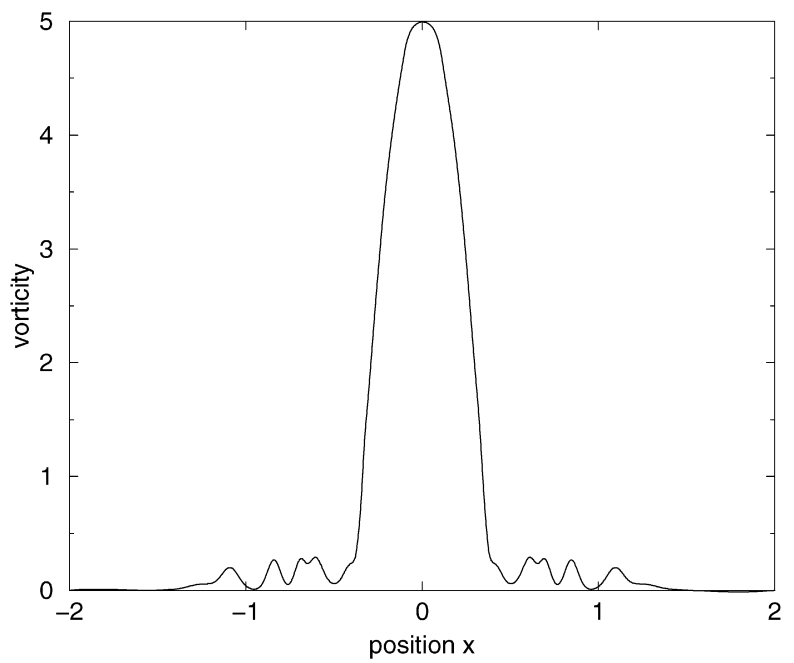

(a)

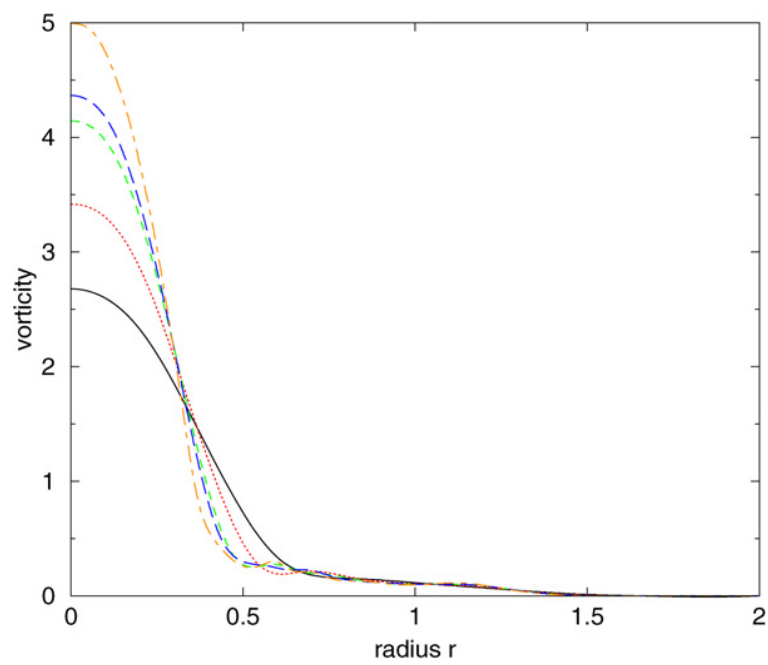

(b)

Fig. 15. (a) Vorticity profile $\omega(r)$ at $t^{*}=t_{f}^{*}$ along the line corresponding to the larger elliptic momentum axis including the filaments at $R e=10000$; (b) The axisymmetric component $\omega_{0}(r)$ of the central vortex at $t^{*}=t_{f}^{*}$ for various Reynolds numbers $R e=1000, R e=15000, R e=20000$, $R e=50000, R e=100000$ from bottom to top.

considered independent of the Reynolds number. Eventual weak correction in $\log (R e)$ cannot be asserted or denied at this numerical precision. The central vortex which is mainly axisymmetric, nevertheless contains an azimuthal dependence in the form of Kelvin waves (see Fig. 16) propagating upon the axisymmetric profile $\omega_{0}(r)$. Note that such waves are similar to those observed before merging when the initial condition does not correspond to a quasiequilibrium state. The wave amplitude (see Fig. 16) decrease in a similar manner for the two Reynolds number presented which indicates that the final vortex profile is not far from being Gaussian [5]. The Gaussian character of the central vortex at $t_{f}^{*}$ has been in fact checked for various Reynolds numbers: the width of the fitted Gaussian is found to slightly decrease with the Reynolds number. 


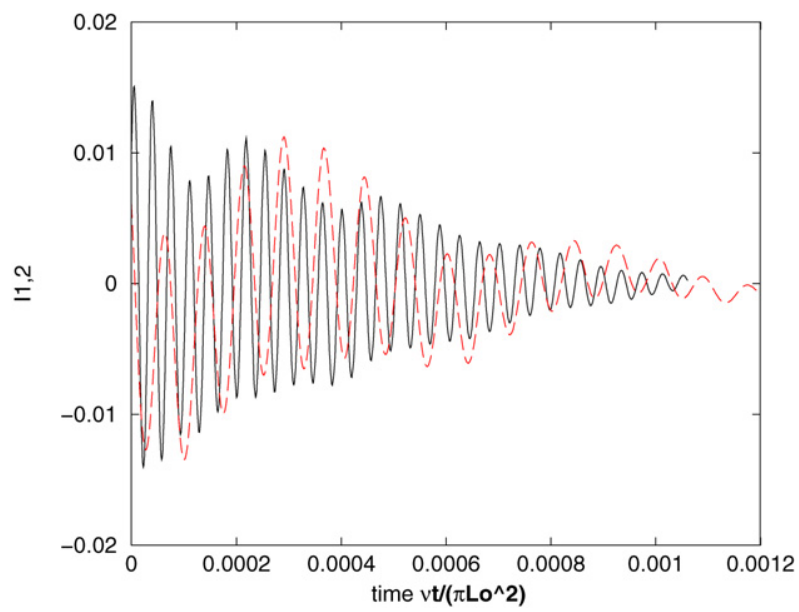

Fig. 16. Elliptical moment $I_{1,2}$ as a function of a viscous times scale $v t / \pi L_{0}^{2}$ (for this case only the origin time $t=0$ have been translated and it corresponds to the time of merging $t^{*}=t_{f}^{*}$ ) for two Reynolds numbers: $R e=30000$ (continuous line), $R e=15000$ (dashed line). These oscillations are the Kelvin waves propagating upon on the axisymmetric profile $\omega_{0}(r)$.

\section{Conclusion}

The merging of a corotating pair of equal vortices was numerically studied for a large range of Reynolds numbers (from 1000 to 100000). The previous method used to pinpoint the merging onset was investigated showing its deficiency and an alternative method was proposed based on the existence of a quasi-equilibrium state before onset. During the rapid phase of merging we measured the contributions of the different flow regions to the relative motion of the two vortex centroids, indicating that the intermediate region between filaments and vortices plays a role as important as the filaments. In the following plateau regime, a scaling in $R e^{1 / 2}$ was identified for the plateau duration and interpreted in terms of an advection-diffusion process. Finally, the structure of the merging outcome was analysed as a function of Reynolds number. We found that the total circulation lost in the filaments was almost independent of Reynolds numbers.

\section{References}

[1] M.J. Shelley, D.I. Meiron, S.A. Orszag, Dynamical aspects of vortex reconnection of perturbed ant-parallel vortex tubes, J. Fluid Mech. 246 (1993) 613-652.

[2] O. Agullo, A.D. Verga, 'Exact two vortices solutions of Navier-Stokes equations, Phys. Rev. Lett. 78 (1997) 2361.

[3] P. Meunier, Etude expérimentale de deux tourbillons corotatifs, PhD Thesis of the université De Provence Aix-Marseille I, IRPHE, 2001.

[4] P. Meunier, U. Ehrenstein, T. Leweke, M. Rossi, A merging criterion for two-dimensional co-rotating vortices, Phys. Fluids 14 (8) (2002) $2757-2766$.

[5] S. Le Dizès, A. Verga, Viscous interactions of two co-rotating before merging, J. Fluid Mech. 467 (2002) 389-410.

[6] C. Cerretelli, C.H.K. Williamson, The physical mechanism for vortex merging, J. Fluid Mech. 475 (2003) 41-77.

[7] C. Cerretelli, C.H.K. Williamson, A new family of uniform vortices related to vortex configurations before merging, J. Fluid Mech. 493 (2003) 219-229.

[8] R.R. Trieling, O.U. Velasco Fuentes, G.J.F. van Heijst, Interaction of two unequal corotating vortices, Phys. Fluids 17 (2005) 087103.

[9] J.C. McWilliams, The vortices of two-dimensional turbulence, J. Fluid Mech. 219 (1990) 361-385.

[10] P. Tabeling, S. Burkhart, O. Cardoso, H. Willaime, Experimental study of freely decaying two-dimensional turbulence, Phys. Rev. Lett. 67 (1991) 3372.

[11] J. Jimenez, H.K. Moffatt, C. Vasco, The structure of the vortices in freely decaying two-dimensional turbulence, J. Fluid Mech. 313 (1996) 209-222.

[12] G.L. Brown, A. Roshko, On density effects and large structure in turbulent mixing layers, J. Fluid Mech. 64 (1974) $775-816$.

[13] A. Chen, J.D. Jacob, O. Savas, Dynamics of corotating vortex pair in the wakes of flapped airfoils, J. Fluid Mech. 382 (1999) $155-193$.

[14] W.J. Devenport, C.M. Vogel, J.S. Zoldos, Flow structure produced by the interaction and merger of a pair of co-rotating wing-tip vortices, J. Fluid Mech. 394 (1999) 357-377.

[15] A. Provenzale, Transport by coherent barotropic vortices, Annu. Rev. Fluid Mech. 31 (1999) 55-93.

[16] R.W. Griffiths, E.J. Hopfinger, Coalescing of geostrophic vortices, J. Fluid Mech. 178 (1987) 73-97.

[17] T. Leweke, C.H.K. Williamson, Cooperative elliptic instability of a vortex pair, J. Fluid Mech. 360 (1998) 85-119. 
[18] P. Meunier, T. Leweke, Three-dimensional instability during vortex merging, Phys. Fluids 13 (2001) 2747-2750.

[19] L. Ting, C. Tung, Motion and decay of a vortex in a nonuniform stream, Phys. Fluids 8 (1965) 1039.

[20] S. Le Dizès, Non-axisymmetric vortices in two-dimension, J. Fluid Mech. 406 (2000) 175-198.

[21] P.G. Saffman, R. Szeto, Equilibrium shapes of a pair of equal uniform vortices, Phys. Fluids 23 (1980) 2339.

[22] D.G. Dritschel, The stability and energetics of corotating uniform vortices, J. Fluid Mech. 157 (1985) 95.

[23] D.G. Dritschel, A general theory for two-dimensional vortex interactions, J. Fluid Mech. 293 (1995) 269.

[24] P.G. Saffman, Vortex Dynamics, Cambridge University Press, Cambridge, 1992.

[25] E.A. Overman, N.J. Zabusky, Evolution and merger of isolated vortex structures, Phys. Fluids 25 (1982) 1297-1305.

[26] U. Ehrenstein, M. Rossi, Equilibria of corotating nonuniform vortices, Phys. Fluids 11 (1999) 3416.

[27] E.A. Overman, Steady-state solutions of the Euler equations in two dimensions. II. Local analysis of limiting V-states, SIAM J. Appl. Math. 46 (1986) 765 .

[28] U. Ehrenstein, S. Le Dizès, Relationship between corotating vortex-pair equilibria and a single vortex in an external deformation field, Phys. Fluids 17 (2005) 074103.

[29] N.K.R. Kevlahan, M. Farge, Vorticity in two-dimensional turbulence: creation, stability and effect, J. Fluid Mech. 346 (1997) $49-76$.

[30] D.G. Dritschel, N.J. Zabusky, On the nature of vortex interactions and models in unforced nearly-inviscid two-dimensional turbulence, Phys Fluids 8 (5) (1996) 1252-1256.

[31] M.V. Melander, N.J. Zabusky, J.C. McWilliams, Symmetric vortex merger in two dimensions: causes and conditions, J. Fluid Mech. 195 (1988) 305-340.

[32] G.F. Carnevale, J.C. McWilliams, Y. Pomeau, J.B. Weiss, W.R. Young, Evolution of vortex statistics in two-dimensional turbulence, Phys. Rev. Lett. 66 (1991) 2735.

[33] For the FFTW routines, see the web site: www.fftw.org.

[34] M. Melander, J.C. McWilliams, N.J. Zabusky, Axisymmetrization and vorticity gradient intensification of an isolated vortex through filamentation, J. Fluid Mech. 178 (1987) 137-159.

[35] A.J. Bernoff, J.F. Lingevitch, Rapid relaxation of an axisymmetric vortex, Phys. Fluids 6 (11) (1994) 3717-3723.

[36] K. Bajer, A. Bassom, A.D. Gilbert, Accelerated diffusion in the centre of a vortex, J. Fluid Mech. 437 (2001) $395-411$. 